\title{
SCHWARTZ DISTRIBUTIONS ANALYTIC IN A PARAMETER AND ASSOCIATED SEMI-ANALYTIC FUNCTIONS
}

\author{
CLIFFORD A. LONG ${ }^{1}$
}

1. Introduction. The notion of distributions depending on a parameter was introduced by Laurent Schwartz and is indicated in the pamphlet on his Canadian lectures written by Israel Halperin [3, p. 17]. Gel'fand and Šilov [2, p. 147] studied the properties of generalized functions depending on a parameter, particularly when the dependence is analytic in the sense indicated below. We show that restrictions placed on distributions over a region $Z$ imply certain associated functions; in particular (Theorem 3), that analytic distributions have associated semi-analytic functions.

2. Basic definitions and results. Let $F(I)$ denote a continuous linear functional on a space $K_{I}$ of testing functions $\phi$ with support contained in an open interval $I$, and let $F(\phi)$ be the corresponding real numbers. The properties of distributions depending on a parameter are in many ways analogous to those of ordinary functions through use of the basic completeness theorem of Schwartz [4, p. 74]: If a sequence of distributions $F_{i}$ is such that, for each $\phi$ in $K, F_{i}(\phi) \rightarrow F(\phi)$, then these numbers $F(\phi)$ determine a distribution $F$.

If $z$ is a complex parameter taking on values in a region $Z$ and for each $z$ there exists a corresponding distribution $F(I ; z)$ with support in $I$, we call $F(I ; z)$ a distribution on $I$ over $Z . F_{0}(I)$ is the limit of $F(I ; z)$ as $z \rightarrow z_{0}$ iff the ordinary function values $F(\phi, z)$ approach $F_{0}(\phi)$ for every $\phi$ in $K_{I}$ as $z \rightarrow z_{0}$. Continuity, differentiability and analyticity of $F(I ; z)$ with respect to $z$ are defined in the standard way using this limit.

$F(I ; z)$ is said to be identified with the point function $f$ iff for each closed bounded interval $R$ in $I, F(\phi, z)=\int_{R} f(x, z) \phi(x) d x$ for each $\phi$ in $K_{R}$ and each $z$ in $Z$. For a closed bounded interval $R, F(R ; z)$ is associated with $f$ iff there is an integer $r \geqq 0$ such that for each $\phi$ in $K_{r}$ and each $z$ in $Z, F(\phi, z)=\int_{R} f(x, z) \phi^{(r)}(x) d x$. If $f$ is continuous in $(x, z)$ and analytic in $z$ throughout $I \times Z$, then $f$ is semi-analytic over $I \times Z$. (This definition is due to Maxime Bôcher who demonstrated properties of such functions [1].)

Received by the editors March 26, 1965.

1 The results of this paper are contained in the author's University of Illinois thesis which was supervised by Pierce W. Ketchum. The research was supported in part by the National Science Foundation. 
If for each closed bounded interval $R$ in $I, F(R ; z)$ is associated with a semi-analytic function, then $F(I ; z)$ is an analytic distribution on $I$ over $Z$. That functions associated with analytic distributions need not be semi-analytic follows from the fact that $f$ is only determined to within an arbitrary polynomial $P$ in $x$ of degree less than $r$, i.e., $F(\phi, z)=\int_{R} f(x, z) \phi^{(r)}(x) d x=\int_{R}[f(x, z)+P(x, z)] \phi^{(r)}(x) d x$, where the coefficients of $P$ may be nonanalytic functions of $z$.

\section{Distributions over $Z$ and associated functions.}

THEOREM 1. If $F(I ; z)$ is a continuous distribution over a closed bounded region $Z$, then for each closed bounded interval $R$ in $I$, there exists an associated $f$ which is uniformly continuous over $R \times Z$.

Proof. (A modification of one given by Schwartz [3, p. 14] which relies on his results.) For each $R=[a, b]$ in $I$, there exists an associated $g$ bounded on $R \times Z$ such that $F(\phi, z)=\int_{R} g(x, z) \phi^{(s)}(x) d x$. Integration by parts yields $F(\phi, z)=\int_{R} h(x, z) \phi^{(s+1)}(x) d x$ where $h(x, z)$ $=-\int_{a}^{x} g(t, z) d t$ is continuous in $x$ on $R$ uniformly over $Z$. Also $F(\phi, z)$ $=\int_{R}[h(x, z)+P(x, z)] \phi^{(s+1)}(x) d x$, with $P$ an arbitrary polynomial in $x$ of degree less than $s+1$. The following lemma completes the proof of the theorem.

LeMma 1. Let $F(I ; z)$ be a continuous distribution over closed region $Z$, and for each closed bounded interval $R$ in $I$, let there be an associated function, $h$, continuous in $x$ on $R$ uniformly over $Z$ such that $F(\phi, z)$ $=\int_{R} h(x, z) \phi^{(r)}(x) d x$. Then there exists a polynomial $P$ in $x$ of degree less than $r$ with bounded functions of $z$ as coefficients, such that: $h(x, z)$ $+P(x, z)=f(x, z)$ defines a bounded function over $R \times Z$, continuous in $x$ on $R$ uniformly over $Z$, continuous in $z$ on $Z$ uniformly over $R$, and associated with $F(I ; z)$.

Proof outline of Lemma 1 . The proof is by induction on $r$. For the case $r=0$, we need to show that $h(x, z)$ is continuous in $z$ on $\bar{Z}$ uniformly over $R$, or, what is equivalent, that $g(x, z)=h(x, z)-h\left(x, z_{0}\right)$ converges to 0 uniformly over $R$ as $z \rightarrow z_{0}$, for each $z_{0}$ in $\bar{Z}$. For each $\epsilon>0$, there exists a $\delta$ such that, for all $z$ in $\bar{Z},|g(x, z)-g(y, z)|<\epsilon$, if $|x-y|<\delta$. Let the closed bounded interval $R=[a, b]$ be covered by a finite number of intervals $I_{1}, I_{2}, \cdots, I_{t}$, each of length $<\delta$. We now show that there exists a neighborhood $N_{0}$ of $z_{0}$ in which, for each $I_{p}$, there is at least one $x_{p}$ in $I_{p}$ such that $g\left(x_{p}, z\right)<\epsilon$ for all $z$ in $N_{0}$. If such an $N_{0}$ did not exist, then we could find a sequence of nested neighborhoods $N_{i}$ of $z_{0}$ with diameters $\rightarrow 0$, such that for each $N_{i}$, there exist an $I_{p}=(c, d)$ and a $z_{i}$ in $N_{i}$ with $g\left(x, z_{i}\right) \geqq \epsilon$ for each $x$ 
in $I_{p}$. For the function with values $\phi_{p}(x)=\exp (1 /(x-d)+1 /(c-x))$ on $I_{p}$ and 0 elsewhere, we would then have $G\left(\phi_{p}, z_{i}\right)=\int_{a}^{b} g\left(x, z_{i}\right) \phi_{p}(x) d x$ $\geqq \epsilon \int_{c}^{d} \phi_{p}(x) d x=\alpha>0$. This contradicts the fact that $G(\phi, z)=F(\phi, z)$ $-F\left(\phi, z_{0}\right)$ converges to 0 . Since there exist only a finite number of $I_{p}$ 's, even if we allowed the $I_{p}$ to vary for different $z_{i}$ 's, we would still obtain a contradiction. Hence $N_{0}$ exists.

Thus, for each $x$ in $R$ and all $z$ in $N_{0},|g(x, z)|=\mid\left[g(x, z)-g\left(x_{p}, z\right)\right]$ $-g\left(x_{p}, z\right) \mid<2 \epsilon$ and $g(x, z)$ converges to 0 uniformly on $R$ as $z \rightarrow z_{0}$.

Suppose that the lemma holds for $r=p$ and consider the distribution $F(R ; z)$ with $F(\rho, z)=\int_{R} h(x, z) \rho^{(p-1)}(x) d x$ where $h$ is continuous in $x$ on $R$ uniformly over $Z$. For each $\rho$ in $K_{R}$, and a fixed $\theta$ in $K_{R}$ with $\int_{R} \theta(x) d x=1$, there exists a function $\phi$ in $K_{R}$, such that $\rho^{\prime}(x)=\phi(x)$ $-a_{0} \theta(x)$, where $a_{0}=\int_{R} \phi(x) d x$. The identity, $\int_{R} h(x, z) \rho^{(p+1)}(x) d x$ $=\int_{R}\left(h(x, z)+b(z) x^{p}\right) \phi^{(p)}(x) d x$, where $b$ is a bounded function of $z$ dependent on $\theta$ and on $g$, but not on $\phi$ is known [3, p. 15]. The form, $G(\phi, z)=\int_{R}\left[h(x, z)+b(z) x^{p}\right] \phi^{(p)}(x) d x$, determines a distribution on $R$ over $Z$. It is continuous over $Z$, because if $\phi$ is fixed, then $G(\phi, z)$ $=F(\rho, z)$ where $\rho(x)=\int_{a}^{x}\left[\phi(x)-a_{0} \theta(x)\right] d x$ and $F(\rho, z)$ is continuous over $Z$ by hypothesis. The case $r=p$ implies the existence of a polynomial $Q$ in $x$ of degree $<p$ with bounded functions of $z$ as coefficients, such that $h(x, z)+b(z) x^{p}+Q(x, z)$ is bounded over $R \times Z$, continuous in $x$ on $R$ uniformly over $Z$ and continuous in $z$ on $Z$ uniformly over $R$. The polynomial, $P$, defined by $P(x, z)=b(z) x^{p}$ $+Q(x, z)$, satisfies the conditions of the lemma for $r=p+1$.

TheOREM 2. Let $F(I ; z)$ be a continuous distribution over a region $Z$ and its boundary. Then $F(I ; z)$ is analytic over $Z$ iff for each closed bounded interval $R$ in $I$, there exists an associated $f$ continuous on $R \times Z$ such that for each closed contour $C$ in $Z, \int_{C} f(x, z) d z$ equals a polynomial in $x$ of degree less than $r$ (in general depending on $C$ ).

Proof (ONLy IF). For $C$ a closed contour in $Z, \int_{C} F(\phi, z) d z=0$, for each $\phi$ in $K_{I}$ by Cauchy's integral theorem. By Theorem 1, for each closed bounded interval $R$ in $I$, there exists an associated $f$ continuous on $R \times Z$. Hence,

$$
\int_{C} F(\phi, z) d z=\int_{C} \int_{R} f(x, z) \phi^{(r)}(x) d x d z=\int_{R} \int_{C} f(x, z) d z \phi^{(r)}(x) d x .
$$

Since $\int_{C} f(x, z) d z=g(x)$ describes a continuous function of $x$, and $\int_{R} g(x) \phi^{(r)} d x=0$ for all $\phi$ in $K_{R}$, we have that $g(x)$ is a polynomial of degree less than $r$ in $x$. It may depend on $C$.

(IF). For each $\phi$ in $K_{R}$ and any closed contour $C$ in $Z, \int_{C} F(\phi, z) d z$ $=\int_{C} \int_{R} f(x, z) \phi^{(r)}(x) d x d z=\int_{R} \int_{C} f(x, z) d z \phi^{(r)}(x) d x=0$, since $\int_{C} f(x, z) d z$ 
is a polynomial in $x$ of degree less than $r$. By Morera's Theorem, $F(\phi, z)$ is analytic over $Z$ for each $\phi$ in $K_{R}$. Since each $\phi$ in $K_{I}$ is in some $K_{R}, F(\phi, z)$ is analytic over $Z$ for each $\phi$ in $K_{I}$, i.e., $F(I ; z)$ is an analytic distribution over $Z$.

CoROllary. If $F(I ; z)$ is an analytic distribution over $Z$ identified with the function $f$ continuous over $I \times Z$, then $f$ is semi-analytic over $I \times Z$.

THeOREM 3. Let $F(I ; z)$ be a distribution over a region $Z$ and its boundary. Then $F(I ; z)$ is analytic over region $Z$ and continuous on its boundary iff for each closed bounded interval $R$ in $I$, there exists an associated semi-analytic function.

Proof (IF). Straightforward.

(ONLY IF) Let $F(I ; z)$ be analytic over region $Z$ and continuous on its boundary. Then by Theorem 2 , for each closed bounded interval there exists an associated function $h$ continuous on $R \times \bar{Z}$. This $h$ satisfies the conditions of Lemma 1 . By induction we show that the polynomial $P$ of the lemma can be chosen so that $f=h+P$ will be continuous over $R \times \bar{Z}$ and analytic over $Z$.

For the case $r=0, f=h$, and hence is already continuous over $R \times \bar{Z}$. That $f$ is also analytic over $Z$ follows from the above corollary to Theorem 2 .

Suppose that a polynomial $P_{p}$ exists for the case $r=p$. To find a polynomial for the case $r=p+1$, we use the method from the proof of Lemma 1 to show that

$$
\int_{R} h(x, z) \rho^{(p+1)}(x) d x=\int_{R}\left(h(x, z)+b(z) x^{p}\right) \phi^{(p)}(x) d x .
$$

Hence, the existence of polynomial $P_{p}$ such that $h(x, z)+b(z) x^{p}+$ $P_{p}(x, z)$ defines a semi-analytic function gives us the required polynomial $Q$ with values $b(z) x^{p}+P_{p}(x, z)$ for the case $r=p+1$.

Thus, for each closed bounded interval $R$ in $I$, there exists an associated semi-analytic function $h+P$.

\section{REFERENCES}

1. M. Bôcher, On semi-analytic functions of two variables, Ann. of Math. (2) 12 (1910-1911), 18-26.

2. I. M. Gel'fand and G. E. Šilov, Generalized functions, Vol. 1. Properties and operations, Academic Press, New York, 1964. Transl. of 1958 Russian ed.

3. I. Halperin, Introduction to the theory of distributions, University of Toronto Press, Toronto, 1952.

4. L. Schwartz, Theorie des distributions, Vol. 1, Actualitiés Sci. Ind. No. 1091, Hermann, Paris, 1950.

Bowling Green State University 